\title{
Production of dijets with large rapidity separation: Mueller-Navelet mechanism versus double-parton scattering
}

\author{
Rafał Maciuła* \\ Institute of Nuclear Physics PAN, PL-31-342 Cracow, Poland \\ E-mail: rafal.maciula@ifj.edu.pl \\ Antoni Szczurek ${ }^{\dagger}$ \\ Institute of Nuclear Physics PAN, PL-31-342 Cracow, Poland and \\ University of Rzeszów, PL-35-959 Rzeszów, Poland \\ E-mail: antoni.szczurek@ifj.edu.pl
}

\begin{abstract}
We discuss hadroproduction of 4-jet final state at the LHC within the double-parton scattering (DPS) mechanism in the context of large-rapidity-distance jets. For planned and/or being currently performed high energy experiments this is the kinematical region where searches for BFKL signal are of the main interest. The DPS contributions are calculated in the LO collinear approach within the so-called factorized Ansatz. We show that the relative contribution of DPS is growing with respect to standard single-parton scattering (SPS) production of dijets and to the BFKL Mueller-Navelet (MN) jet mechanism at large rapidity distance between jets. This is consistent with recent studies of DPS effects in the case of double $D$ and double $J / \psi$ meson production. The calculated differential cross sections as a function of rapidity distance between the jets that are the most distant in rapidity are compared with recent results of LL and NLL BFKL calculations for the Mueller-Navelet jet production at $\sqrt{s}=7 \mathrm{TeV}$. The DPS contribution is carefully studied for $\sqrt{s}=7 \mathrm{TeV}$ and $\sqrt{s}=14 \mathrm{TeV}$ and in different ranges of jet transverse momenta.
\end{abstract}

XXII. International Workshop on Deep-Inelastic Scattering and Related Subjects,

28 April - 2 May 2014

Warsaw, Poland

\footnotetext{
${ }^{*}$ Speaker.

${ }^{\dagger}$ This work was supported in part by the Polish grant DEC-2011/01/B/ST2/04535 as well as by the Centre for Innovation and Transfer of Natural Sciences and Engineering Knowledge in Rzeszów.
} 


\section{Introduction}

About 25 years ago Mueller and Navelet predicted that large-rapidity-distance jets are more decorrellated in azimuth than jets placed close in rapidity [1]. This effect has been related to exchange of the BFKL ladder between outgoing partons. The basic picture is shown in diagram (a) of Fig. 1. The correlation between the most forward and the most backward jets (partons) is expected to be small because of diffusion along the exchanged ladder. Within a simple leading-logarithmic (LL) BFKL formalism (see also e.g. Ref. [2]) quarks/antiquarks/gluons are emitted forward and backward, whereas gluons emitted along the ladder populate rapidity regions in between.

However, this simple picture has been slightly modified by recent next-to-leading logarithmic (NLL) BFKL calculation where the effect of azimuthal decorrelation for large-rapidity-distance jets is predicted to be smaller than in the case of the original expectations (see e.g. Ref. [3] and references therein). On the other hand the dijet azimuthal correlations were also studied in next-toleading order (NLO) collinear approximation [4].

Large-rapidity-distance jets can be measured and/or studied theoretically only at high energies where the rapidity range is large. The LHC opens a possibility to study the decorrelation effect quantitatively at so far not available experimentally distances in rapidity (up to 9.4 units). Until the moment only averaged values of $\left\langle\cos \left(n \phi_{j j}\right)>\right.$ over available phase space or even their ratios were studied experimentally [5]. However, more detailed experimental studies are necessary to verify theoretical predictions. First absolutely normalized experimental cross sections at $\sqrt{s}=7 \mathrm{TeV}$ are expected soon.
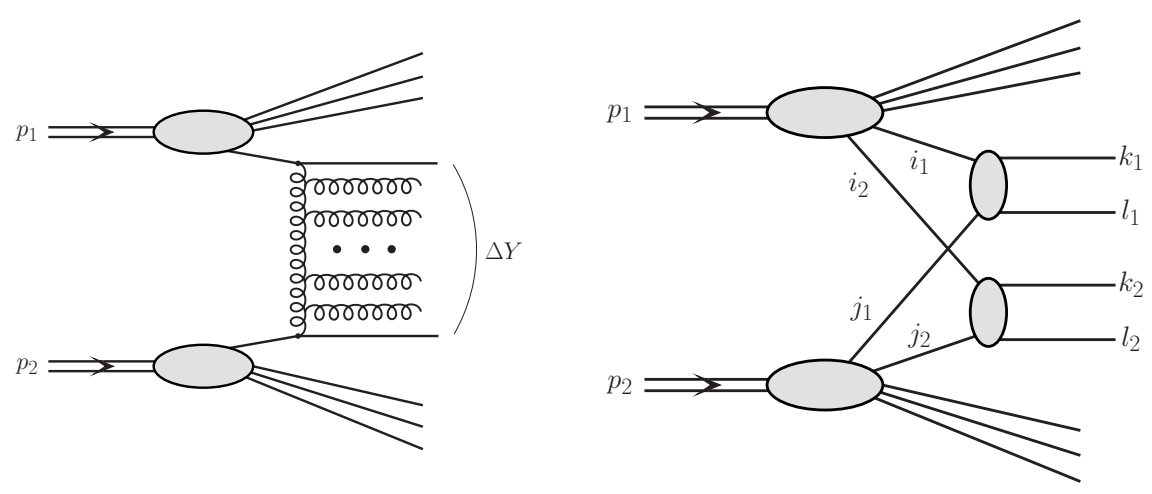

Figure 1: A diagramatic representation of the Mueller-Navelet jet production (left diagram) and of the double parton scattering mechanism (right diagram).

On the other hand recent studies of multiparton interactions have shown that they may easily produce particles (objects) which are emitted far in rapidity. Good example is production of $c \bar{c} c \bar{c}$ $[6,7,8]$ or inclusive production of two $J / \psi$ mesons [9]. Here we wish to concentrate on four-jet double-parton scattering (DPS) production with large distances between jets (see diagram (b) in Fig. 1). Several suggestions how to separate four-jet DPS contribution from SPS contribution at midrapidities were discussed in Ref. [10].

In the present studies, the DPS effects for jets with large rapidity separation are calculated within leading-order (LO) collinear approximation. It has been shown that within this approach even at $\mathrm{LO}$ one gets quite reasonable description of observables for inclusive jet production at 
the LHC [11] and therefore may be use for an exploratory estimation of the DPS contribution to the considered reaction. The numerical result for large-rapidity-distance jets within the DPS mechanism will be compared to the distribution in rapidity distance for collinear SPS PQCD as well as for the BFKL Mueller-Navelet dijet calculations.

\section{Basic formalism}

In the present analysis we have taken into account all partonic cross sections $(i j \rightarrow k l)$ which are calculated in LO collinear approximation. The cross section for dijet production can be written then as:

$$
\frac{d \sigma(i j \rightarrow k l)}{d y_{1} d y_{2} d^{2} p_{t}}=\frac{1}{16 \pi^{2} \hat{s}^{2}} \sum_{i, j} x_{1} f_{i}\left(x_{1}, \mu^{2}\right) x_{2} f_{j}\left(x_{2}, \mu^{2}\right) \overline{\left|\mathscr{M}_{i j \rightarrow k l}\right|^{2}},
$$

where $y_{1}, y_{2}$ are rapidities of the two jets ( $k$ and $\left.l\right)$ and $p_{t}$ is transverse momentum of one of them (they are identical). The parton distributions are evaluated at $x_{1}=\frac{p_{t}}{\sqrt{s}}\left(\exp \left(y_{1}\right)+\exp \left(y_{2}\right)\right)$, $x_{2}=\frac{p_{t}}{\sqrt{s}}\left(\exp \left(-y_{1}\right)+\exp \left(-y_{2}\right)\right)$ and $\mu^{2}=p_{t}^{2}$ is used as factorization and renormalization scale.

According to so-called factorized Ansatz, the DPS differential cross section for jets widely separated in rapidity within the simple LO collinear approach can be written as:

$$
\frac{d \sigma^{D P S}(p p \rightarrow 4 \text { jets } X)}{d y_{1} d y_{2} d^{2} p_{1 t} d y_{3} d y_{4} d^{2} p_{2 t}}=\sum_{\substack{i_{1}, j_{1}, k_{1}, l_{1} \\ i_{2}, j_{2}, k_{2}, l_{2}}} \frac{\mathscr{C}}{\sigma_{e f f}} \frac{d \sigma\left(i_{1} j_{1} \rightarrow k_{1} l_{1}\right)}{d y_{1} d y_{2} d^{2} p_{1 t}} \frac{d \sigma\left(i_{2} j_{2} \rightarrow k_{2} l_{2}\right)}{d y_{3} d y_{4} d^{2} p_{2 t}}
$$

where $\mathscr{C}=\left\{\begin{array}{l}\frac{1}{2} \text { if } i_{1} j_{1}=i_{2} j_{2} \wedge k_{1} l_{1}=k_{2} l_{2} \\ 1 \text { if } i_{1} j_{1} \neq i_{2} j_{2} \vee k_{1} l_{1} \neq k_{2} l_{2}\end{array}\right\}$ and partons $i, j, k, l=g, u, d, s, \bar{u}, \bar{d}, \bar{s}$. The combinatorial factors include identity of the two simultaneous subprocesses. The quantity $\sigma_{e f f}$ is responsible for a proper normalization of the DPS events, has dimension of cross section and has a simple interpretation in the impact parameter representation [12]. Above $y_{1}, y_{2}$ and $y_{3}, y_{4}$ are rapidities of partons (jets) in "first" and "second" partonic subprocess, respectively. The $p_{1 t}$ and $p_{2 t}$ are respective transverse momenta.

In our numercial calculation in the denominator of formula in Eq.(2.2) we take $\sigma_{\text {eff }}=15 \mathrm{mb}$ which is world average value extracted from the Tevatron and the LHC data for different processes. A detailed analysis of the $\sigma_{e f f}$ parameter based on various experimental data can be found e.g. in Ref. [13].

\section{Numerical Results}

In Fig. 2 we show distribution in the rapidity distance between two jets in the standard SPS dijet collinear calculation and between jets that are the most distant in rapidity in the case of four DPS jets. In this calculation we have included cuts characteristic for the CMS experiment: $y_{1}, y_{2} \in$ (-4.7,4.7), $p_{1 t}, p_{2 t} \in(35 \mathrm{GeV}, 60 \mathrm{GeV})$. For comparison we show also results for the LL and NLL BFKL calculation for MN jets from Ref. [3]. For this kinematics the DPS jets give rather large relative contribution to two jet case only at the largest rapidity distances. The NLL BFKL cross section (long-dashed line) is smaller than that for the SPS pQCD dijet mechanism (short-dashed line). Our DPS contribution seems to be of the same order of magnitude as the NLL BFKL, and therefore is potentially important. 


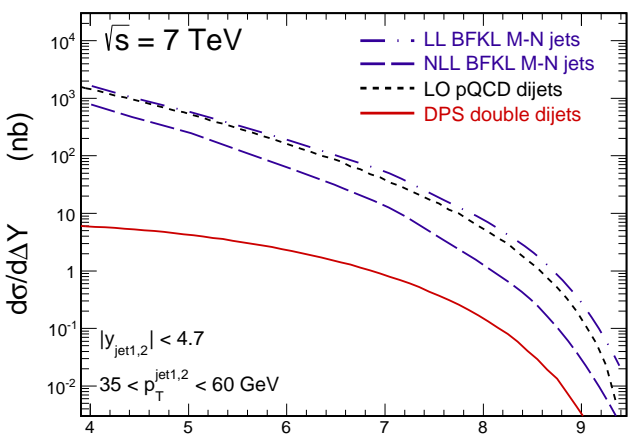

$\Delta \mathrm{Y}$

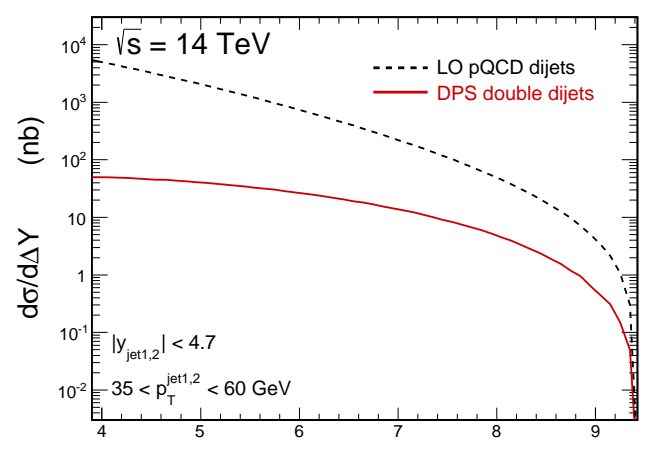

$\Delta \mathrm{Y}$

Figure 2: Distribution in rapidity distance between jets $\left(35 \mathrm{GeV}<p_{t}<60 \mathrm{GeV}\right.$ ) with maximal (the most positive) and minimal (the most negative) rapidities. The collinear pQCD result is shown by the short-dashed line and the DPS result by the solid line. The calculation has been performed for $\sqrt{s}=7 \mathrm{TeV}$ (left panel) and $\sqrt{s}=14 \mathrm{TeV}$ (right panel). For comparison we show also results for the BFKL Mueller-Navelet jets in leading-logarithm and next-to-leading-order logarithm approaches taken from Ref. [3].

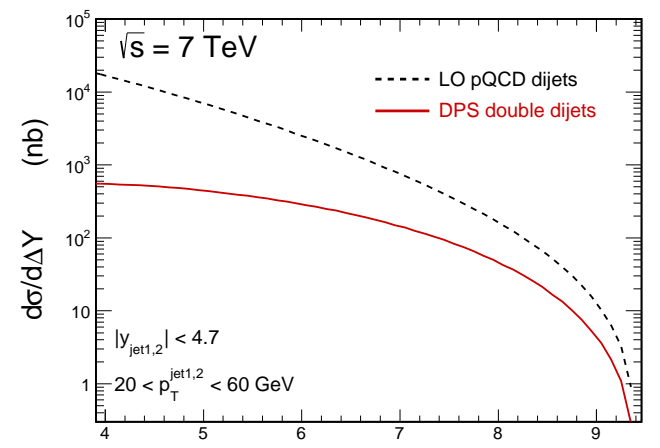

$\Delta \mathrm{Y}$

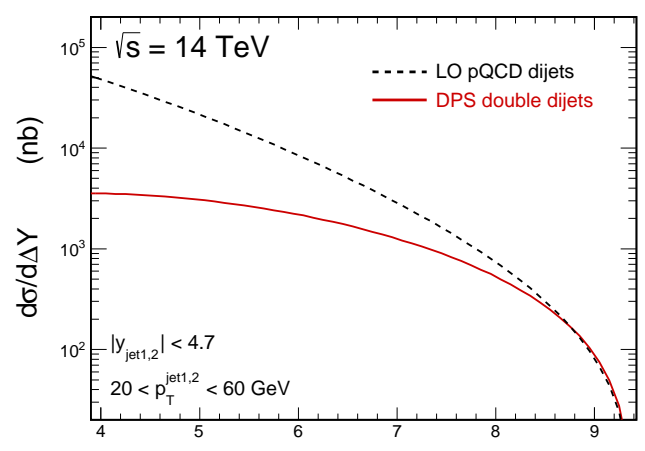

$\Delta \mathrm{Y}$

Figure 3: The same as in the previous figure but now for somewhat smaller lower cut on jet transverse momentum, $p_{t}>20 \mathrm{GeV}$.

As for the BFKL Mueller-Navelet jets the DPS contribution is growing with deacreasing jet transverse momenta. In Fig. 3 we show rapidity-distance distribution for even smaller lowest limit for transverse momentum of the jet, i.e. $p_{t}>20 \mathrm{GeV}$. Now the relative contribution of the DPS mechanism with respect to the standard SPS PQCD dijet production become meaningly larger and certainly cannot be neglected. We have checked that further lowering of the jet $p_{t}$ lower limit leads to the situation where the DPS contribution even exceeds the standard SPS one. However, measurement of such (mini)jets may be rather difficult. In principle, one could measure for instance correlations of semihard $\left(p_{t} \sim 10 \mathrm{GeV}\right)$ neutral pions with the help of so-called zero-degree calorimeters (ZDC) which are installed by all major LHC experiments. Other possibilities could also be considered. 


\section{Conclusions}

We have discussed how the double-parton scattering effects may contribute to large-rapiditydistance dijet correlations and whether they potentially could or not shadow the BFKL signal from the Mueller-Navelet jets.

We have shown that the contribution of the DPS mechanism increases with increasing distance in rapidity between jets. For the CMS configuration the DPS contribution is smaller than the standard SPS dijet mechanism even at the highest energy and at high rapidity distances but only slightly smaller than that for the NLL BFKL calculation known from the literature. A contamination of the large-rapidity-distance jets by the DPS effects may distort the information on genuine Mueller-Navelet jets, especially at the nominal LHC energy, and make the comparison with the BFKL calculation not fully conclusive. A detailed analysis of this contamination will be a subject of our future studies.

The DPS final state topology is clearly different than that for the SPS dijets which may help to separate the two mechanisms experimentally. Of course SPS three- and four-jet final states should be included in more detailed analyses.

We have shown also that the relative effect of DPS could be increased by lowering of the transverse momenta of jets but such measurements can be difficult. Alternatively one could study correlations of semihard pions that are most distant in rapidity. This type of studies requires a dedicated Monte Carlo analyses taking into account also hadronization effects.

\section{References}

[1] A. H. Mueller, and H. Navelet, Nucl. Phys. B282, 727 (1987).

[2] V. Del Duca and C. R. Schmidt, Phys. Rev. D49, 4510 (1994).

[3] B. Ducloue, L. Szymanowski and S. Wallon, J. High Energy Phys. 05, 096 (2013).

[4] P. Aurenche, R. Basu and M. Fontannaz, Eur. Phys. J. C57, 681 (2008).

[5] S. Chatrchyan et al. (the CMS Collaboration), CMS-PAS-FSQ-12-002 (2013).

[6] M. Łuszczak, R. Maciuła, and A. Szczurek, Phys. Rev. D85, 094034 (2012).

[7] R. Maciuła, and A. Szczurek, Phys. Rev. D87, 074039 (2013).

[8] A. van Hameren, R. Maciuła and A. Szczurek, arXiv:1402.6972 [hep-ph].

[9] S. P. Baranov et al., Phys. Rev. D87, 034035 (2013).

[10] E. L. Berger, C. B. Jackson and G. Shaughnessy, Phys. Rev. D81, 014014 (2010).

[11] R. Maciuła and A. Szczurek, arXiv:1403.2595 [hep-ph] (in print in Physical Review D).

[12] C. Flensburg, G. Gustafson, L. Lönnblad and A. Ster, J. High Energy Phys. 06, 066 (2011).

[13] M. H. Seymour, and A. Siódmok, J. High Energy Phys. 10, 113 (2013); arXiv:1307.5015 [hep-ph]. 